\title{
Research on Communication Networks Effectiveness Evaluation Based on AHP and Fuzzy Clustering
}

\author{
Ding Yan \\ State grid Qinhuangdao Power Supply Company, Qinhuangdao, China \\ vicacer1@163.com
}

Keywords: Analytic hierarchy process(AHP), Fuzzy clustering, Communication networks effectiveness, Evaluation

\begin{abstract}
Communication networks effectiveness evaluation directly determined whether the communication networks system is successful. This paper investigated the communication networks effectiveness evaluation based on Analytic hierarchy process and fuzzy clustering method. Indicators of communication networks effectiveness are established, and the weights of them are obtained by analytic hierarchy process. After that, sample data for a communication networks is gathered by practical research. Then these data is clustered by fuzzy clustering method. Finally, this communication networks is evaluated by four classes including excellent, good, qualified and failure. This research explored an effective method for communication networks effectiveness evaluation.
\end{abstract}

\section{Introduction}

Communication network effectiveness evaluation is a practical problem, but also a theoretical problem. It is the foundation of establishing high reliable network information system and the prerequisite of improving survivability and survivability of communication network [1]. It can be said that communication network effectiveness evaluation is directly related to the success or failure of communication network system. At present, there are few studies on the effectiveness evaluation of communication networks, mainly focusing on the research of technical problems in the design stage, which limits the improvement of the actual network operation reliability level [2]. Because the communication network system has the complex structure and the technology is diverse, the different type network's influence exerts the influence factor to be different [3]. Therefore, it is very important to select the suitable appraisal index system, use the reasonable mathematical modeling method, determine each appraisal target weight and evaluate the effectiveness of the communication network accurately. Therefore, this paper proposes a method to evaluate the effectiveness of communication network and fuzzy clustering analysis model by combining analytic hierarchy process and fuzzy clustering analysis, and explore a reasonable and reliable evaluation method for communication network efficiency.

\section{Communication Networks Effectiveness Evaluation Index System Establishment}

As the structure of communication networks is diverse, the technologies adopted by different networks are not the same as the services they undertake. Therefore, the selection of effectiveness evaluation index system is also different. In order to select the appropriate indicators from the complex factors and correctly reflect the overall effectiveness of the network, it is necessary to analyze the function and structure of the communication network, and consider the special use of all aspects, taking into account the comprehensive effectiveness. Here we learn from previous studies, and build a communication network effectiveness evaluation index system, as shown in Fig. 1.

As shown in Fig. 1, the effectiveness of the communication network is evaluated by four aspects of evaluation index system, namely, response capability, processing capability, effectiveness and reliability. For the four indicators, we consider using expert scoring methods to determine the corresponding points, namely, very good (4 points), good (3 points), qualified (2 points), basic qualified (1 point) and poor ( 0 point). The system response capacity is mainly based on response 
time, delay and delay jitter three factors to score. The system processing capacity is mainly based on throughput, call loss rate, channel bandwidth, bit error rate and signal to noise ratio and other five factors to score. The system reliability is mainly based on the channel connectivity and node utilization to score. The system reliability is mainly based on end connectivity reliability, network connectivity reliability, connectivity and cohesion of four factors to score.

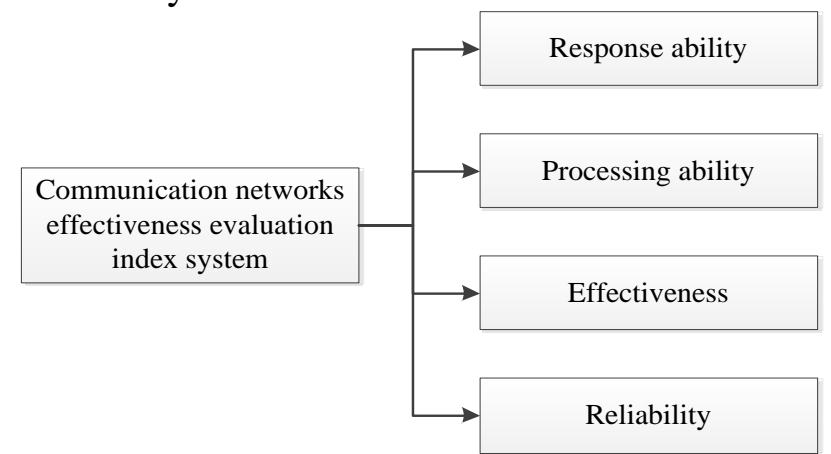

Fig. 1 Evaluation index system of communication networks effectiveness

The basic idea of communication network effectiveness evaluation is shown in Fig. 2. Firstly, according to the evaluation index system of communication network effectiveness, Analytic hierarchy process (AHP) is used to determine the influence weights of each index on the effectiveness of communication network. On this basis, the weight of the sample data is processed. Finally, fuzzy cluster analysis is used to analyze the effectiveness of communication network. The clustering analysis is performed on the weighted sample data, and the effectiveness evaluation of the communication network is given.

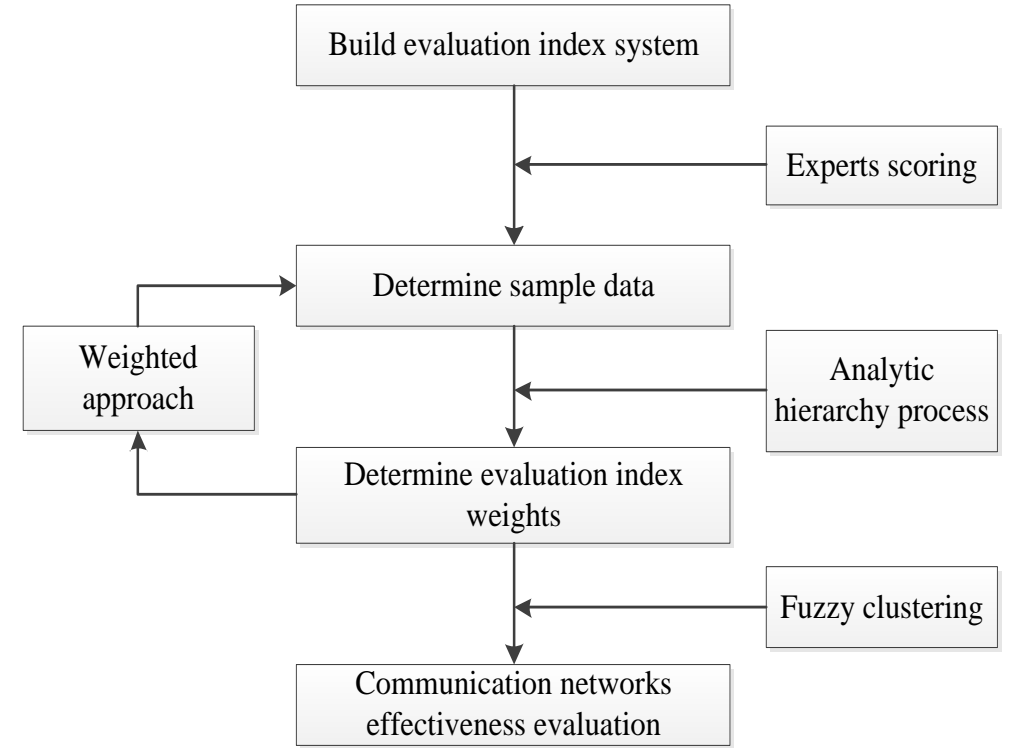

Fig. 2 The basic idea of communication network effectiveness evaluation

\section{Determine the Weights of Evaluation Indexes Using AHP}

AHP is a decision-making method which decomposes elements related to decision-making into objectives, criteria and schemes, and then qualitative and quantitative analysis. Its implementation process mainly includes the following five steps: (1) establishing the hierarchical structure model; (2) constructing the judgment matrix; (3) determining the consistency; (4) the overall ranking of the hierarchy; (5) the consistency of the overall ranking of the test[4].

It is not difficult to find that the evaluation indexes given in Fig. 1 are different for the influence of communication network effectiveness. Using AHP is convenient to determine the impact weight of these indexes on the effectiveness of communication network. By comparing the importance of each index, we establish AHP judgment matrix p as shown in Table 1. 
Table 1 Judgment matrix

\begin{tabular}{|c|c|c|c|c|}
\hline Scale & $\begin{array}{c}\text { Response } \\
\text { ability }\end{array}$ & $\begin{array}{c}\text { Processing } \\
\text { ability }\end{array}$ & Effectiveness & Reliability \\
\hline Response ability & 1 & 3 & 7 & 5 \\
\hline Processing ability & $1 / 3$ & 1 & 5 & 7 \\
\hline Effectiveness & $1 / 7$ & $1 / 5$ & 1 & $1 / 3$ \\
\hline Reliability & $1 / 5$ & $1 / 7$ & 3 & 1 \\
\hline
\end{tabular}

According to the judgment matrix p, calculate the impact weights of each index, as shown in Table 2. Then, use consistency testing to judge the reasonableness of weights.

Table 2 Index weight

\begin{tabular}{|c|c|c|c|c|}
\hline Index & $\begin{array}{c}\text { Response } \\
\text { ability }\end{array}$ & $\begin{array}{c}\text { Processing } \\
\text { ability }\end{array}$ & Effectiveness & Reliability \\
\hline Weight & 0.316 & 0.293 & 0.190 & 0.201 \\
\hline
\end{tabular}

The result of consistency ranking shows that there is a satisfactory consistency in the results of hierarchical ranking, that is, the performance evaluation index of communication network affects the reasonable distribution of weights.

\section{Fuzzy Clustering Analysis of Communication Networks Effectiveness Evaluation}

Fuzzy clustering method is an extension of cluster analysis. In this paper, the dynamic clustering method based on fuzzy equivalence relation is used to evaluate the efficiency of communication network [5]. The basic implementation process is divided into five steps: pretreatment of sample data, establishment of fuzzy similar matrix, dynamic clustering analysis, optimal the classification result and communication network performance evaluation. The basic process is shown in Fig. 3.

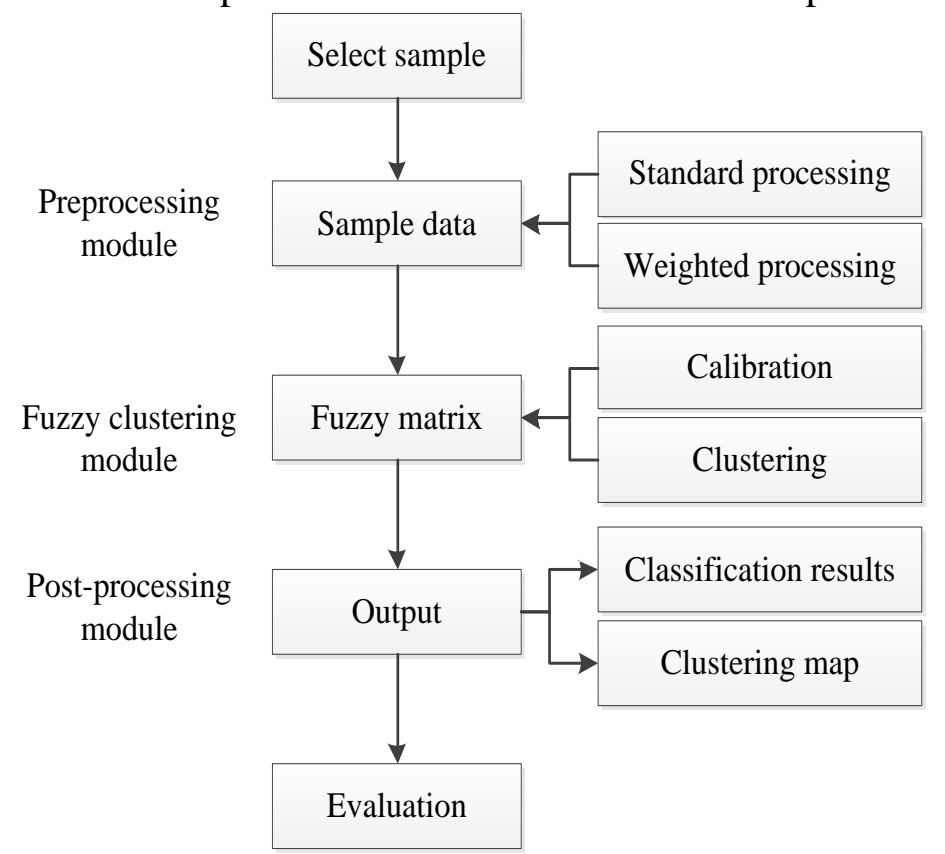

Fig. 3 Fuzzy clustering evaluation model

Determine Sample and Data Preprocessing. In order to evaluate the effectiveness of the communication network based on the fuzzy equivalence relation clustering analysis method, we use the evaluation index system established above to investigate and analyze the actual effectiveness of a communication system, and use the expert scoring method to determine the 12 sample data , shown in Table 3.

Before the fuzzy clustering analysis, we need to standardize the sample data. Standardization is divided into two steps:

Dimensionless, the formula is as follow: 


$$
x_{i j}^{\prime}=\frac{x_{i j}-\bar{x}_{j}}{s_{j}}
$$

Table 3 Actual data

\begin{tabular}{|c|c|c|c|c|}
\hline No. & $\begin{array}{c}\text { Response } \\
\text { ability }\end{array}$ & $\begin{array}{c}\text { Processing } \\
\text { ability }\end{array}$ & Effectiveness & Reliability \\
\hline 1 & 4 & 1 & 0 & 2 \\
\hline 2 & 3 & 3 & 2 & 4 \\
\hline 3 & 4 & 4 & 3 & 1 \\
\hline 4 & 2 & 1 & 2 & 0 \\
\hline 5 & 1 & 4 & 1 & 1 \\
\hline 6 & 3 & 1 & 0 & 4 \\
\hline 7 & 2 & 2 & 3 & 3 \\
\hline 8 & 2 & 0 & 4 & 1 \\
\hline 9 & 4 & 2 & 4 & 4 \\
\hline 10 & 3 & 3 & 1 & 1 \\
\hline 11 & 1 & 2 & 0 & 3 \\
\hline 12 & 2 & 3 & 4 & 0 \\
\hline
\end{tabular}

Where $\bar{x}_{j}$ represents the average value of $j$ index, $s_{j}$ is standard deviation of $j$ index.

Extreme normalization, the data are compressed in the $[0,1]$, the formula is as follow:

$$
x_{i j}^{\prime \prime}=\frac{x_{i j}^{\prime}-\left\{x_{i j}^{\prime}\right\}_{\min }}{\left\{x_{i j}^{\prime}\right\}_{\max }-\left\{x_{i j}^{\prime}\right\}_{\min }}
$$

Where $\left\{x_{i j}^{\prime}\right\}_{\max }$ and $\left\{x_{i j}^{\prime}\right\}_{\min }$ respectively represent the maximum and minimum values in the normalized values.

Build Fuzzy Similarity Matrix. Based on the weighting of classification index, this paper uses the correlation coefficient method to calculate the fuzzy similarity matrix of samples. The formula is:

$$
\gamma_{i j}=\frac{\sum_{k=1}^{n}\left(x_{i k}-\bar{x}_{i}\right)\left(x_{j k}-\bar{x}_{j}\right)}{\sqrt{\sum_{k=1}^{n}\left(x_{i k}-\bar{x}_{i}\right)^{2}} \sqrt{\sum_{k=1}^{n}\left(x_{j k}-\bar{x}_{j}\right)^{2}}}
$$

Where $\bar{x}_{i}$ and $\bar{x}_{j}$ respectively represent the average value of $i$ and $j$ sample after standardized treatment, $n$ is the number of indexes.

According to the calibration formula of correlation coefficient method, the weighted fuzzy similarity matrix $R$ can be obtained which is shown in Table 4 .

Table 4 Fuzzy similarity matrix

\begin{tabular}{|c|c|c|c|c|c|c|c|c|c|c|c|c|}
\hline No. & 1 & 2 & 3 & 4 & 5 & 6 & 7 & 8 & 9 & 10 & 11 & 12 \\
\hline 1 & 1.00 & 0.54 & 0.02 & 0.25 & 0.28 & 0.24 & 0.17 & 0.37 & 0.17 & 0.39 & 0.27 & 0.40 \\
\hline 2 & 0.54 & 1.00 & 0.66 & 0.62 & 0.54 & 0.55 & 0.47 & 0.49 & 0.44 & 0.25 & 0.35 & 0.27 \\
\hline 3 & 0.02 & 0.66 & 1.00 & 0.81 & 0.76 & 0.83 & 0.79 & 0.82 & 0.76 & 0.38 & 0.43 & 0.46 \\
\hline 4 & 0.25 & 0.62 & 0.81 & 1.00 & 0.86 & 0.80 & 0.61 & 0.90 & 0.57 & 0.62 & 0.23 & 0.62 \\
\hline 5 & 0.28 & 0.54 & 0.76 & 0.86 & 1.00 & 0.89 & 0.82 & 0.93 & 0.81 & 0.79 & 0.06 & 0.90 \\
\hline 6 & 0.24 & 0.55 & 0.83 & 0.80 & 0.89 & 1.00 & 0.94 & 0.95 & 0.93 & 0.74 & 0.11 & 0.79 \\
\hline 7 & 0.17 & 0.47 & 0.79 & 0.61 & 0.82 & 0.94 & 1.00 & 0.86 & 1.00 & 0.66 & 0.14 & 0.76 \\
\hline 8 & 0.37 & 0.49 & 0.82 & 0.90 & 0.93 & 0.95 & 0.86 & 1.00 & 0.85 & 0.78 & 0.05 & 0.80 \\
\hline 9 & 0.17 & 0.44 & 0.76 & 0.57 & 0.81 & 0.93 & 1.00 & 0.85 & 1.00 & 0.67 & 0.19 & 0.77 \\
\hline 10 & 0.39 & 0.25 & 0.38 & 0.62 & 0.79 & 0.74 & 0.66 & 0.78 & 0.67 & 1.00 & 0.56 & 0.90 \\
\hline 11 & 0.27 & 0.35 & 0.43 & 0.23 & 0.06 & 0.11 & 0.14 & 0.05 & 0.19 & 0.56 & 1.00 & 0.45 \\
\hline 12 & 0.40 & 0.27 & 0.46 & 0.62 & 0.90 & 0.79 & 0.76 & 0.80 & 0.77 & 0.90 & 0.45 & 1.00 \\
\hline
\end{tabular}


Dynamic Clustering Analysis. The so-called cluster is based on the fuzzy equivalence matrix $R^{*}$, with different threshold $\lambda$ for interception, so as to obtain different classification. The fuzzy similarity matrix $R$, which has been established above, only satisfies reflexivity and symmetry in general, and does not satisfy the transitivity. Thus, it is not a fuzzy equivalence relation. To this end, we need to transform $R$ into a fuzzy equivalent matrix. Use the square method to find the transport package $t(R)$ of $R$. After four operations $R \rightarrow R^{2} \rightarrow R^{4} \rightarrow R^{8} \rightarrow R^{16}$, so $t(R)=R^{*}=R^{16}=R^{8}$. When $\lambda$ is 0.82 , the samples are clustered into seven categories; When $\lambda$ is 0.75 , the samples are clustered into five categories; When $\lambda$ is 0.67 , the samples are clustered into four categories; When $\lambda$ is 0.56 , the samples are clustered into two categories; When $\lambda$ is 0.53 , the samples are clustered into one category.

Communication Network Effectiveness Evaluation. Since the samples are clustered into different classes when $\lambda$ is taken at different levels, we need to determine an optimal partition, which can be analyzed by the $F$ statistic. $F$ is calculated as:

$$
F=\frac{\sum_{j=1}^{r} n_{j} \sum_{k=1}^{m} \frac{\left(\bar{x}_{k}^{(j)}-\bar{x}_{k}\right)^{2}}{r-1}}{\sum_{j=1}^{r} \sum_{i=1}^{n_{j}} \sum_{k=1}^{m} \frac{\left(x_{i k}^{(j)}-\bar{x}_{k}^{(j)}\right)^{2}}{n-r}}
$$

Where $r$ is the total number of classes, and $n$ is the total number of samples.

If $\mathrm{F}>\mathrm{F} 0.05$, according to mathematical statistics analysis of variance theory, the difference between classes and significant, indicating a more reasonable division. The results show that $\lambda=$ $0.67, \mathrm{~F}=0.05$ is the maximum of all classes, and the samples are classified into four categories, namely, class I $\{1,3,9\}$, class II $\{2,6,10\}$, class III $\{4,8,12\}$, class IV $\{5,7,11\}$. According to this, we evaluate the effectiveness of the communication network by four grades: excellent, good, qualified and unqualified.

\section{Conclusion}

In this paper, for the complexity of the communication network system structure, analytic hierarchy process and fuzzy clustering method are applied to analyze the effectiveness of communication network evaluation. Through the construction of the communication network efficiency evaluation index system, and then based on the analytic hierarchy process to determine the impact of each indicator weights. By analyzing the actual effectiveness of a communication system, a certain amount of sample data is obtained and a fuzzy clustering analysis method is used to evaluate the effectiveness of the communication network successfully, namely, excellent, good, qualified and unqualified. The research of this paper explores an effective method for the effectiveness evaluation of communication network, and achieves good results.

\section{References}

[1] Lu Ziyi, Fan Jianhua. Effectiveness Evaluation of Tactical Communication Network Based on AHP[J]. Modern Electronics Technique, 2011, 34(1): 57-60.

[2] Wu Chao, Guo Xiaolei. Study on the Effectiveness Evaluation Method of Military Communication Network Based on BP Neutral Network [J]. Journal of CAEIT, 2016, 11(3): 300-304.

[3] Ren Shuting, Guo Lili. An Evaluation Method based on Cloud Neural Network for HF Communication Effectiveness [J]. Communications Technology, 2014, 47(2), 195-199.

[4] Deng Xue, Li Jiaming, Zeng Haojian, Chen Junyang, Zhao Junfeng. Research on Computation Methods of AHP Weight Vector and Its Applications [J]. Mathematics In Practice and Theory, 2012, 42(7): 93-100. 
[5] Zhang Min, Yu Jian. Fuzzy Partitional Clustering Algorithms [J]. Journal of Software, 2004, 15(6): 858-868. 